\title{
On the Security of PAS (Predicate-based Authentication Service)
}

\author{
Shujun $\mathrm{Li}^{*}$, Hassan Jameel Asghar ${ }^{\dagger}$, Josef Pieprzyk ${ }^{\dagger}$, Ahmad-Reza Sadeghi ${ }^{\ddagger}$, Roland Schmitz ${ }^{\S}$ and Huaxiong Wang ${ }^{\Uparrow}$ \\ ${ }^{*}$ Department of Computer and Information Science, University Konstanz, Germany \\ ${ }^{\dagger}$ Center for Advanced Computing - Algorithms and Cryptography, Macquarie University, Australia \\ ${ }^{\ddagger}$ System Security Group, Ruhr-University of Bochum, Germany \\ $\S$ Department of Computer Science and Media, Stuttgart Media University, Germany \\ IDivision of Mathematical Sciences, Nanyang Technological University, Singapore
}

\begin{abstract}
Recently a new human authentication scheme called PAS (predicate-based authentication service) was proposed, which does not require the assistance of any supplementary device. The main security claim of PAS is to resist passive adversaries who can observe the whole authentication session between the human user and the remote server.

In this paper we show that PAS is insecure against both brute force attack and a probabilistic attack. In particular, we show that its security against brute force attack was strongly overestimated. Furthermore, we introduce a probabilistic attack, which can break part of the password even with a very small number of observed authentication sessions. Although the proposed attack cannot completely break the password, it can downgrade the PAS system to a much weaker system similar to common OTP (one-time password) systems.
\end{abstract}

Keywords-PAS; authentication; Matsumoto-Imai threat model; attack; security; usability; OTP (one-time password);

\section{Introduction}

An important and foremost requirement of every security system is user authentication. A user authentication method enables a system to give access to legitimate users while denying access to impersonators. Roughly speaking, user authentication methods can be divided into the following basic three categories according to how the verifier authenticates a user: 1) "what you know" - via a secret shared between the legitimate user and the verifier; 2) "what you possess" - via a physical token the user possesses; 3 ) "who you are" - via an inherent characteristic of the user. Typical examples of user authentication systems belonging to the above three categories include password-based systems ("what you know"), smart card based systems ("what you possess") and biometrics-based systems ("who you are").

Different user authentication methods are designed to be secure under different threat models. One important threat model involves adversaries who can eavesdrop on or even tamper communications between the user and the verifier. Apparently, fixed passwords are not secure under this threat model, since they can be simply recorded and replayed later by an adversary to impersonate the protected identities. As possible solutions, dynamic passwords like one-time passwords (OTP) or more complicated challengeresponse user authentication protocols have to be adopted.

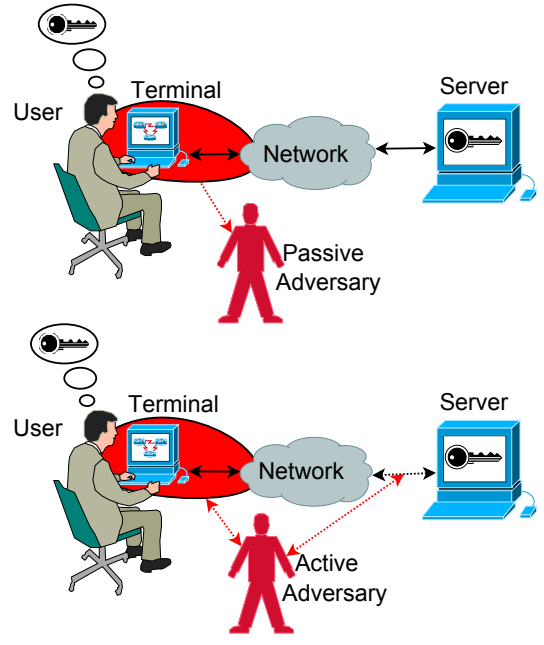

Figure 1. Matsumoto-Imai threat model: the red area shows the information source available to the adversaries, and the arrows denote information flow.

To assist human users to calculate the one-time passwords or correct responses to dynamic challenges, some specialpurpose hardware/software is often a must.

In a stronger threat model described by Matsumoto \& Imai in [1], it is assumed that the human user does not have access to any special-purpose hardware/software. Instead, the only resource a human user can use is his/her own brain. There are two types of adversaries in this threat model passive and active adversaries as shown in Figure 1. Passive adversaries can observe all the user's interaction with the terminal and/or all the communications between the terminal and the remote server. In comparison, active adversaries can further modify the communications between the terminal (i.e., the user) and the remote server. A lot of practical attacks belong to or have close link to the above threat model, such as shoulder-surfing attack, key/screen-logger attack, phishing/pharming attack, malware-based attack, manin-the-middle attack, and so forth. In the literature, the term "observer attack", "observation attack" and "peeping attack" are also used to cover attacks under this threat model [2].

Generally speaking, a secure user authentication system under Matsumoto-Imai threat model is a challenge-response 
protocol based on a secret shared between the user and the server. The user has to make correct responses to a number of challenges dynamically generated by the server to prove his/her identity. There are several design goals of such a challenge-response user authentication protocol:

1) Usability: the correct response to each challenge is easy for a legitimate user to calculate mentally.

2) Security against passive adversaries: it is computationally infeasible to derive the secret or part of it from a number of observed authentication sessions.

3) Security against active adversaries: it is computationally infeasible to choose some challenges to ease the derivation of the secret or part of it.

Since the 1990s there have been a number of attempts at designing user authentication systems which are secure against passive adversaries, which will be introduced in Section II. A recent design was predicate-based authentication service (PAS) proposed in [3], which was designed to resist passive adversaries. In this paper, we show that the original security claims given by Bai et al. in [3] are not correct. A probabilistic attack is proposed to partially break the secret shared between the user and the server, which downgrades the PAS scheme to a much weaker authentication system.

The rest of the paper is organized as follows. Some related work is introduced in the next section. Then, we briefly describe how the PAS scheme works in Section III. A reevaluation of security and usability of the PAS scheme is given in Section IV, and a probabilistic attack is proposed in Section V. The last section concludes the paper.

\section{Related Work}

To the best of our knowledge, the earliest attempt was made by Matsumoto and Imai after they introduced the threat model [1]. Wang et al. showed that the Matsumoto-Imai protocol was not secure enough against active adversaries [4]. Wang et al. also proposed a modified scheme, but its usability is too low for common users in practice. In [5] Matsumoto proposed several new protocols based on the dot product of two vectors. According to [2], these dot-productbased protocols are not sufficiently secure against passive adversaries, in the sense that the secret can be revealed with a linear (in the size of the secret) number of observed authentication sessions (which was also pointed out in [6]).

In [7] $\mathrm{Li}$ and Teng proposed a new protocol based on lexical shifting and matching. No cryptanalysis was reported on Li-Teng protocol, but its usability is doubtful since the user has to remember three different kinds of secrets, each of which is of a considerable length.

Two protocols based on hard mathematical problems were proposed by Hopper and Blum in [6]. The main problem with Hopper-Blum protocols is again about usability: the password has to be long enough to ensure security, which makes usability relatively low. One Hopper-Blum protocol also requires the user to make intentional errors with probability $\eta$, which may not be an easy task for many common users. According to the user study on a prototype system reported in [6], the average login time is around 160 seconds, which may be too long for a practical system.

In [8], Li and Shum suggested some principles and two general structures of designing challenge-response protocols secure under Matsumoto-Imai threat model, which are based on making balanced errors and hiding direct responses to challenges, respectively. A graphical implementation was designed. No cryptanalysis has been reported on this work, but the usability of the graphical implementation is also questionable, since the login time is considerably long.

Jameel et al. proposed a new image-based solution [9] and shortly after extended it for devices with limited display [10]. This solution is based on a hidden rule classifying images into two categories. One problem with this design is how the server collects images in different categories, since the hidden rule is generally not executable by a computer. If the image classification task has to be done manually by the user, it is doubtful if the solution can offer an acceptable balance between usability and security.

In [11] Weinshall proposed two new solutions based on image recognition capabilities of humans. However, Golle and Wagner showed that both solutions are insecure against SAT (satisfiability solver) attack [12]. This attack is very effective, since it requires only a small number of observed authentication sessions. The usability of these solutions is also questionable, since the user has to remember $30-80$ pictures, which may not be an easy task even with training.

Besides the above proposed solutions, there is also quite a lot of work aiming at the weakest type of passive adversaries - shoulder surfers [13]-[18]. The main goal is to avoid password leaking from a few number of authentication sessions observed by a shoulder surfer. Since the security level is considerably relaxed, it becomes much easier to design practical solutions secure against shoulder surfers.

While most designs try to hide the password or correct responses from being observed by attackers, recently Sasamoto et al. proposed to hide part of the challenges [19]. In this specific design called UnderCover, the hidden challenge is realized in a way such that the user's palm resting on a haptic device obscures any external observation. While this solution does not ask the user to bring any special-purpose hardware, the terminal equipped with the haptic device has to be trustable, which cannot be ensured in some real attacks.

The main difficulty of designing a user authentication protocol secure under Matsumoto-Imai threat model is to find an acceptable balance between security and usability. Many solutions can be made secure by merely increasing the password size, but this makes the systems unusable in practice. Another noticeable difficulty is the imbalance between the human users and the potential adversaries. While human users can depend only on their brains, adversaries generally 


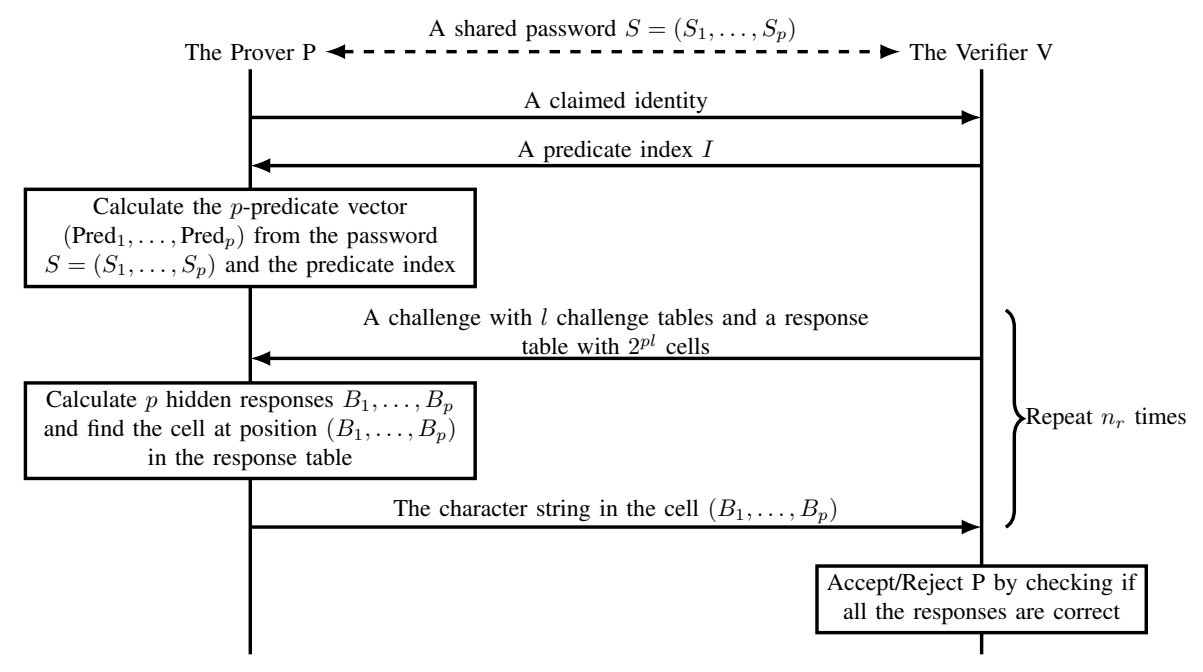

Figure 2. The authentication process of the PAS scheme.

have access to more powerful computational resources.

\section{Introduction to PAS}

In this section, we try to keep the original notations used in [3], but some of them are changed to avoid potential confusion and to maintain consistency among different notations.

In PAS, the prover $\mathrm{P}$ (the human user) and the verifier $\mathrm{V}$ (the PAS server) share a password $S$ composed of $p$ secrets $S_{1}, \ldots, S_{p}$. Each secret $S_{i}$ consists of a 2-D secret cell index $\left(u_{i}, v_{i}\right)$ and a secret word of size len $W_{i}=w_{i}[1] \cdots w_{i}[$ len $]$. The 2-D index denotes a cell at position $\left(u_{i}, v_{i}\right)$ in an $m \times n$ 2-D grid, so $1 \leq u_{i} \leq m$ and $1 \leq v_{i} \leq n$. Each character of the secret word belongs to an alphabet $\mathbb{H}$ of size $H$. Since the 2-D index can be transformed to a 1-D index $c_{i}=\left(u_{i}-1\right)$. $n+v_{i} \in\{1, \ldots, M=m n\}$, in this paper we will analyze the PAS system by replacing $\left(u_{i}, v_{i}\right)$ with the equivalent 1-D index $c_{i} \in\{1, \ldots, M\}$. That is, each secret will be represented as $S_{i}=\left(c_{i}, W_{i}\right)=\left(c_{i}, w_{i}[1] \cdots w_{i}[\right.$ len] $)$. A password with parameter $p=2$, len $=7, M=25$ looks like "(12, catchme; 25 , beathim)".

PAS is a challenge-response protocol, in which the verifier $\mathrm{V}$ raises a number of challenges and the provers $\mathrm{P}$ must give correct responses to all challenges in order to pass the authentication process. To achieve security against passive adversaries, $p$ "predicates" (instead of the password $S$ ) are used to make responses to challenges. The $p$ predicates are dynamically calculated by the prover $\mathrm{P}$ from the secret $S$ and a predicate index $I$, which is sent from $\mathrm{V}$ to $\mathrm{P}$ at the beginning of each authentication session. The prover $\mathrm{P}$ calculates $\hat{I}=(I \bmod l e n)+1$ and generates the $p$ predicates as follows: $i=1, \ldots, p, \operatorname{Pred}_{i}=\left(c_{i}, h_{i}\right)$, where $h_{i}=w_{i}[\hat{I}]$. In this paper, Pred $=\left(\operatorname{Pred}_{i}\right)_{i=1}^{p}$ is called a $p$ predicate vector and also a predicate pair when $p=2$. The predicate pair derived from the password "(12, catchme; 25 , beathim)" and $I=2$ will be "(12, a; 25, e)".

Each challenge raised by the verifier $\mathrm{V}$ includes $l$ challenge tables, each of which contains $M$ cells filled with a certain number of distinct characters in $\mathbb{H}$. To ensure that each character occurs in each cell with probability 0.5 , the number of characters in each cell is always $H / 2$ when $H$ is even, and is $(H-1) / 2$ or $(H+1) / 2$ with probability $\beta=0.5$ when $H$ is odd. In this paper we assume $H$ is even and so each cell always contains $H / 2$ characters. Note that in the default setting of the PAS scheme $H=26$. In addition to the $l$ challenge tables, the verifier $\mathrm{V}$ also sends a $p$ dimensional response table to the prover P. Each dimension of the response table has $2^{l}$ possible values, so there are $2^{p l}$ cells in the response table. All the cells are filled with $2^{l}$ distinct character strings, each of which occurs exactly in $2^{(p-1) l}$ cells. See Figs. 1 and 2 in [3] for examples of the challenge and the response table.

The prover $\mathrm{P}$ constructs a response to each challenge based on the response table and $p$ hidden responses generated from the $p$ predicates. For the $i$-th predicate $\operatorname{Pred}_{i}=$ $\left(c_{i}, h_{i}\right)$, the corresponding hidden response is an $l$-bit integer $B_{i}=b_{i}[1] \cdots b_{i}[l]$, where $b_{i}[j]=1$ if $h_{i}$ occurs in the $c_{i}$ th cell of the $j$-th challenge table and $b_{i}[j]=0$ otherwise. With the $p$ hidden responses, the prover $\mathrm{P}$ finds the cell at the position $\left(B_{1}, \ldots, B_{p}\right)$ in the response table, and sends the character string in that cell as the response to the challenge.

A step-by-step description of the authentication process of the PAS scheme is shown in Fig. 2.

In [3], it is not clearly explained how the predicate index $I$ should be generated. Instead, there is a discussion on the number of authentication sessions (denoted by $t$ ) each predicate index $\hat{I}$ can be used. The maximal number $t_{\max }$ turns out to be 1 for the default setting of the PAS scheme. This means that each possible value of $\hat{I}$ is used for one authentication session only, and the password has to be 
renewed after all the len possible values are exhausted. The predicate indices of the len authentication sessions may simply be chosen as $1, \ldots$, len or a permutation of the len values. In this paper, we assume the PAS scheme runs in a "random permutation mode", in which a random permutation of $1, \ldots$, len determines the predicate index used for each authentication session.

In [3] the above basic PAS scheme is also extended to allow $k>1$ cell indices in each secret $S_{i}$. In this case, the $i$-th secret in the password is redefined as $S_{i}=\left(c_{i, 1}, \ldots, c_{i, k}, W_{i}\right)$. Accordingly, $k$ predicate indices $I_{1}, \ldots, I_{k}$ will be sent from $\mathrm{V}$ to $\mathrm{P}$ for each authentication session. The prover $\mathrm{P}$ calculates the $i$-th predicate $\operatorname{Pred}_{i}$ as a set of $k$ sub-predicates $\left\{\operatorname{Pred}_{i, j}\right\}_{j=1}^{k}$, where $\operatorname{Pred}_{i, j}=$ $\left(c_{i, \hat{I}_{j, k}}, h_{i, j}\right), h_{i, j}=w_{i}\left[\hat{I}_{j, l e n}\right], \hat{I}_{j, k}=\left(I_{j} \bmod k\right)+1$ and $\hat{I}_{j, l e n}=\left(I_{j} \bmod l e n\right)+1$. With this extended predicate containing $k$ sub-predicates, the hidden response $B_{i}$ of the $i$-th predicate is obtained as follows: the prover $\mathrm{P}$ first calculates $k$ hidden sub-responses $B_{i, 1}, \ldots, B_{i, k}$ for the $k$ sub-predicates in the same way as in the basic PAS scheme, and then determines $B_{i}$ as the bitwise OR of the $k$ hidden sub-responses: $B_{i}=B_{i, 1} \vee \cdots \vee B_{i, k}$. To ensure uniform distribution of $B_{i}$ over $\left\{0, \ldots, 2^{l}-1\right\}$, the number of distinct characters in each cell of each challenge table and the corresponding probability $\beta$ should be determined by Eqs. (6) and (8) in [3], respectively.

A list of the parameters and notations involved in the description of the PAS scheme is given in Table I. The default parameters used in [3] are: $p=2$, len $=10$, $\mathbb{H}=\{A, \ldots, Z\}$ (so $H=26), l=2, M=25, n_{r}=5$, $k=1$. We omit notations of the extended scheme (except for $k$ ) to save space. All of them can be obtained by extending the basic notations as described above.

In [3], the security of the PAS scheme was analyzed against three different possible attacks: brute force attack, random guess attack and SAT (satisfiablity solver) attack. Three different attack targets were checked: password, predicate, and response. By assuming each predicate index is used for $t$ authentication sessions, the security was measured in term of the cardinality of the attack set, i.e., the size of the reduced target space, or the number of candidate targets passing all the observed authentication sessions. Table II shows the results reported in [3]. By setting a minimal security level for each possible attack, Bai et al. also described how to get $t_{\max }$, the maximal number of authentication sessions a predicate index $\hat{I}$ can be repeatedly used. For the default setting of the basic PAS scheme, it was claimed that $t_{\max } \approx 1$ so that the same password $S$ can be used for at least $t_{\max } \cdot$ len $=10$ times before renewal.

In [3] a usability study is also reported for a prototype system with the default parameters and $n_{r}=2,3,4,5$. The average time consumed on deriving the predicates from secrets was around 35 seconds, and that for each challenge
Table I

LIST OF PARAMETERS/NOTATIONS USED IN THE DESCRIPTION OF PAS.

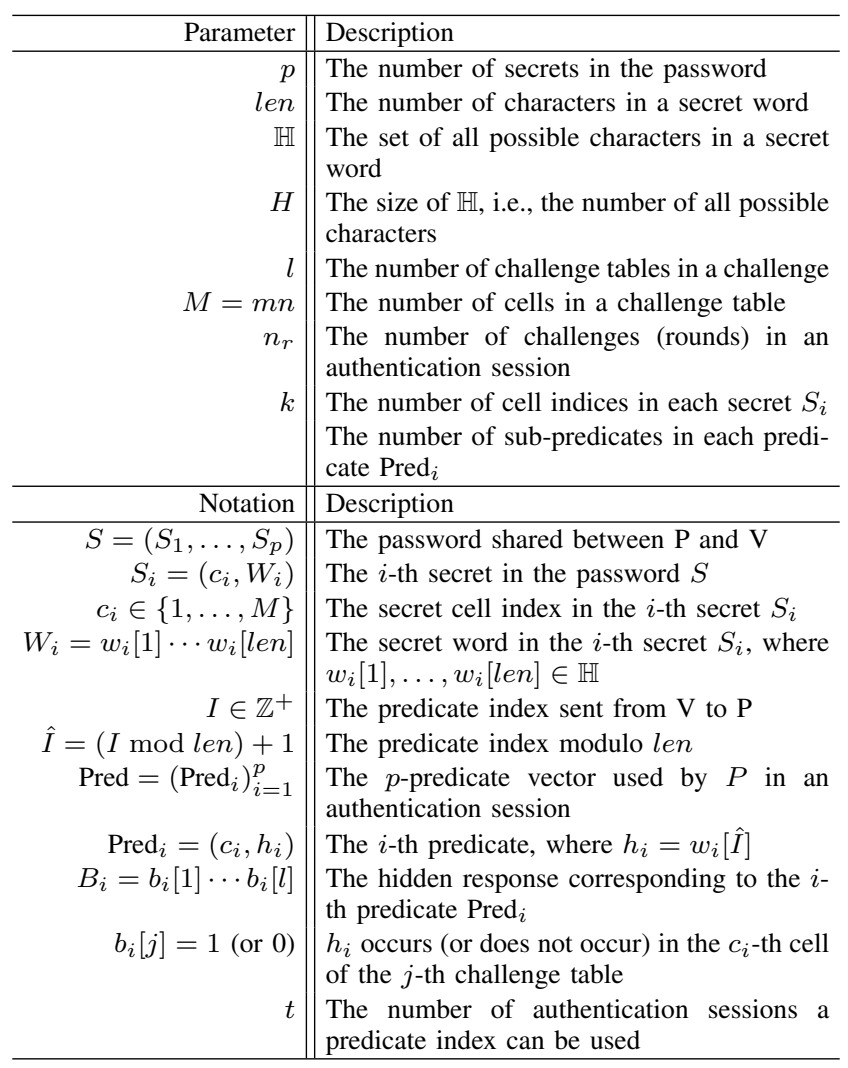

round ranged from 8.37 to 10.5 seconds. When $n_{r}=5$, the total login time for one authentication session was around 84 seconds on average. A survey on the upper bound of the login time was also conducted, and more than half of the participants chose 2 minutes. We will use these statistical data to discuss the relationship between security and usability of the PAS scheme.

\section{Security and Usability of PAS}

First of all, the definitions of two of the three attacks in [3] are problematic. In Table II, there are two "NA"-s for brute force attack, and security against brute force attack is the same as security against random guess attack. In fact, according to the definitions given in [3], the brute force attack and the random guess attack are actually the same attack if the target is the password.

In our opinion, the brute force attack should be defined as exhaustively searching the whole password/predicate space $\mathbb{S}$ to determine a subspace (i.e., an "attack set" according to the term used in [3]) $\mathbb{S}^{*} \subseteq \mathbb{S}$, which is composed of all candidates of the password/predicate that pass all the authentication sessions observed by a passive adversary. Apparently, the correct password/predicate used by the human prover $\mathrm{P}$ is always in the subspace $\mathbb{S}^{*}$. When $\left|\mathbb{S}^{*}\right|=1$ or 
Table II

THE SECURITY OF PAS AGAINST THREE DIFFERENT ATTACKS, REPORTED IN TABLE 1 OF [3].

\begin{tabular}{c||c|c|c}
\hline & Password & Predicate & Response \\
\hline Brute Force & $M^{p k} H^{p \cdot l e n}$ & NA & NA \\
\hline Random Guess & $M^{p k} H^{p \cdot l e n}$ & $(M H)^{p k} /(k !)^{p}$ & $2^{l n_{r}}$ \\
\hline \multirow{2}{*}{ SAT } & $\begin{array}{c}\left(M\left(1-\left(1-\frac{1}{M}^{N}\right)^{l e n / k}\right)^{p k} H^{p \cdot l e n},\right. \\
\text { where } N=p k(M H)^{p k} /\left(2^{l n_{r} t}(k !)^{p}\right)\end{array}$ & $\left(M\left(1-\left(1-\frac{1}{M}\right)^{N}\right)^{l e n / k} H\right)^{p k} /(k !)^{p}$ & NA \\
\hline
\end{tabular}

Table III

RE-EVALUATED SECURITY OF PAS AGAINST THREE ATTACKS.

\begin{tabular}{c||c|c|c}
\hline & Password & Predicate & Response \\
\hline Brute Force / SAT & $\left(1+\left(\left(\begin{array}{c}M H+k-1 \\
k\end{array}\right)^{p}-1\right) / 2^{l n_{r} t}\right) \frac{l e n !}{(l e n-k) !}$ & $1+\left(\left(\begin{array}{c}M H+k-1 \\
k\end{array}\right)^{p}-1\right) / 2^{l n_{r} t}$ & NA \\
\hline Random Guess & \multicolumn{2}{c|}{$1 /\left(1 / 2^{l n_{r}}+\left(2^{l n_{r}}-1\right) /\left(2^{\ln _{r}}\left(\begin{array}{c}M H+k-1 \\
k\end{array}\right)^{p}\right)\right)<2^{l n_{r}}$} & $2^{l n_{r}}$ \\
\hline
\end{tabular}

small enough, we say the brute force attack is successful.

Just as its name implies, the random guess attack should be defined as randomly guessing the correct password, predicate or response of each challenge in order to pass the authentication session. Note that in the brute force attack the goal is to (maybe partially) reveal the password, but in the random guess attack the goal is to simply impersonate a claimed identity without trying to break any target.

In [3] it is claimed that brute force attack does not take the predicates as the target, because they vary from session to session. We have a different opinion. Since the cell indices remain the same for all predicates, breaking the cell indices (as part of each predicate) may help an attacker pass a later authentication attempt with higher probability before password renewal. Therefore, it is important to consider brute force attack targeting predicates.

In the following, we re-evaluate the security of PAS, and point out that the security of the PAS scheme was overestimated in [3]. Our new estimation is shown in Table III. We also point out the extended PAS scheme is not practical in terms of usability, which allows us to focus only on the basic PAS scheme in the next section.

\section{A. Security against Brute Force Attack Target- ing Predicates}

To facilitate the following discussion, denote the number of distinct $p$-predicate vectors by $N(p, k)$. In [3], the value of $N(p, k)$ was estimated to be $(M H)^{p k} /(k !)^{p}$. Unfortunately, this estimation is wrong. This can be easily verified when $k>1$ and $\operatorname{gcd}(M H, k)=1$. In this case, $(M H)^{p k} /(k !)^{p}$ is not an integer. To derive the correct value of $N(p, k)$, note the following fact: the number of distinct sub-predicates in the $i$-th predicate ranges from 1 to $k$. Thus, we immediately have $N(p, k)=\left(\left(\begin{array}{c}M H \\ 1\end{array}\right)+\left(\begin{array}{c}M H \\ 2\end{array}\right)+\ldots+\left(\begin{array}{c}M H \\ k\end{array}\right)\right)^{p}=$ $\left(\begin{array}{c}M H+k-1 \\ k\end{array}\right)^{p}=\left(\frac{(M H+k-1) \cdots(M H)}{k !}\right)^{p} \geq(M H)^{p k} /(k !)^{p}$.

Although the value of $N(p, k)$ was not overestimated, the influence of $n_{r}$ and $t$ on the size of the attack set was neglected in [3]. However, when the attacker tries to use a randomly selected incorrect $p$-predicate vector to calculate the response to each challenge, the probability of getting the correct response is only $1 / 2^{l}$ (under the assumption that the calculated response has a uniform distribution). Assuming that the responses to different challenges are independent of each other, the probability that a randomly selected predicate will pass $t$ observed authentication sessions will be $1 / 2^{l n_{r} t}$. Since there are one correct $p$-predicate vector and $\left(\begin{array}{c}M H+k-1 \\ k\end{array}\right){ }^{p}-1$ incorrect ones, with $t$ observed authentication sessions the average size of the attack set will be $1+\left(\left(\begin{array}{c}M H+k-1 \\ k\end{array}\right)^{p}-1\right) / 2^{l n_{r} t}$, which is much smaller than the estimation reported in [3]. The computational complexity of the brute force attack is still $O\left(\left(\begin{array}{c}M H+k-1 \\ k\end{array}\right)^{p}\right)$, since all the possible predicates have to be checked one by one.

\section{B. Security against Brute Force Attack Target- ing Password}

When the target of brute force attack is the password $S$, [3] reports that the password space is $M^{p k} H^{p \cdot l e n}$, which is the number of all possible $p$-dimension vectors $\left(S_{1}, \ldots, S_{p}\right)$. However, due to the special design of the PAS scheme, a password $S$ can be equivalently represented as $\frac{l e n !}{(l e n-k) !}$ distinct $p$-predicate vectors: Pred $=\left(\operatorname{Pred}_{i}\right)_{i=1}^{p}$, where $\frac{l e n !}{(l e n-k) !}$ is the number of all possible values of the $k$-tuple predicate-index vector $\left(\hat{I}_{1, l e n}, \ldots, \hat{I}_{k, l e n}\right)$ and $\operatorname{Pred}_{i}=\left(c_{i, \hat{I}_{1, k}}, \ldots, c_{i, \hat{I}_{k, k}}, w_{i}\left[\hat{I}_{1, l e n}\right] \cdots w_{i}\left[\hat{I}_{k, l e n}\right]\right)$. Note that any change in one predicate will not influence any other predicates, so they are independent of each other. As a result, the password space can be calculated as the union of all the predicate spaces. Then, the size of the modified password space is $\left(\begin{array}{c}M H+k-1 \\ k\end{array}\right)^{p} \frac{l e n !}{(l e n-k) !}$, which may be much smaller than $M^{p k} H^{p \cdot l e n}$ in case len $>k$ and $H>l e n$. For the default parameters, Table IV shows how 
Table IV

THE RATIO BETWEEN THE SIZE OF THE RE-REPRESENTED PASSWORD SPACE AND THAT OF THE ORIGINAL PASSWORD SPACE.

\begin{tabular}{c||c|c|c|c|c|c|c|c|c|c}
\hline$k$ & 1 & 2 & 3 & 4 & 5 & 6 & 7 & 8 & 9 & 10 \\
\hline$r$ & 24.5 & 21.3 & 18.5 & 16 & 13.8 & 11.8 & 10.1 & 8.6 & 7.3 & 6.5 \\
\hline
\end{tabular}

the ratio $r=\log _{10}\left(M^{p k} H^{p \cdot l e n} /\left(\left(\begin{array}{c}M H+k-1 \\ k\end{array}\right)^{p} \frac{l e n !}{(l e n-k) !}\right)\right)$ changes as $k$ increases from 1 to len $=10$. We can see $r$ is always much larger than 1, i.e., the size of the rerepresented password space is always much smaller than $M^{p k} H^{p \cdot l e n}$. This can be best demonstrated for the basic PAS scheme. In this case, each password can be represented as len independent predicates, and the password space is reduced to $(M H)^{p} \cdot$ len, which is smaller than $M^{p} H^{l e n \cdot p}$ as long as $H^{l e n \cdot(p-1)}>$ len. For the default parameters, the password space is only $(M H)^{p} \cdot$ len $=(25 \times 26)^{2} \cdot 10 \approx 2^{22}$, which is too small from a cryptographic point of view. Since the cell index for each predicate is always the same, we can separately store the $p$ cell indices $c_{1}, \ldots, c_{p}$ and the len $p$-character words $\left\{W_{j}^{*}=w_{1}[j] \cdots w_{p}[j]\right\}_{j=1}^{l e n}$. Apparently, this is just a reorganization of different parts of the password, so no extra memory is needed.

After representing the password space as the union of $\frac{l e n !}{(l e n-k) !}$ predicate spaces, we can easily obtain the size of the attack set with $t$ observed authentication sessions for each predicate based on the result we obtained in the last subsection. That is $\left(1+\left(\left(\begin{array}{c}M H+k-1 \\ k\end{array}\right)^{p}-1\right) / 2^{l n_{r} t}\right) \frac{l e n !}{(l e n-k) !}$.

\section{Security against Random Guess Attack}

In random guess attack one does not need to try all passwords/predicates/responses, but randomly pick one from the password/predicate/response space and see if he can pass the authentication session. For random guess attack, there is no attack set, but we can use the reciprocal of the success probability of passing the authentication session as an equivalent metric of the security measurement.

When an attacker chooses a random response, the original estimation in [3] is correct, since there are $2^{l}$ possible responses. But the attacker can get a higher success rate if he chooses a random predicate/password. It is because the attacker has a chance to guess the correct predicate/password, which always leads to the correct response. For all the other incorrect predicates, the success rate is the same as that of randomly guessing the response. The overall success rate is

$$
\begin{aligned}
& 1 \cdot \frac{1}{\left(\begin{array}{c}
M H+k-1 \\
k
\end{array}\right)^{p}}+\frac{1}{2^{l n_{r}}} \cdot \frac{\left(\begin{array}{c}
M H+k-1 \\
k
\end{array}\right)^{p}-1}{\left(\begin{array}{c}
M H+k-1 \\
k
\end{array}\right)^{p}}=
\end{aligned}
$$

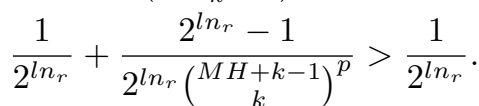

\section{Security against SAT Attack}

The SAT attack can be considered as a special form of brute force attack. Observing our result obtained for brute force attack and the one for SAT attack reported in [3] (when the attack target is the password), one can easily see the former is much smaller than the latter in most cases. For instance, for the basic PAS scheme with the default parameters and $t=1$, the latter is as high as $2^{103.3}$, but the former is only about $2^{22} \ll 2^{103.3}$. This implies that the security analysis on SAT attack given in [3] was also highly over-estimated.

\section{E. Usability}

In [3] it is claimed that the usability of the (basic) PAS scheme is much better than some other solutions (see the last sentence of Section 5.1 of [3]). We doubt if it is a fair comparison. The main problem is the lack of a consistent security analysis of the solutions. The existence of multiple security factors also makes it difficult to find a reasonable parameter set of each solution to compare the usability. For instance, the Cognitive Authentication Scheme (CAS) proposed in [11] has a low-complexity variant, which has relatively good usability but a lower security level according to [12]. Comparing the CAS solution with the default setting of the basic PAS scheme, we have the following results:

- average login time: CAS -1.5 minutes $=90$ seconds, PAS - 84.23 seconds;

- security against random guess attack: CAS $-2^{20} \sim 2^{25}$, PAS - $2^{10}$

- maximal number of authentication sessions a password can be used: CAS - less than 12, PAS - around 10 (actually less, see the next section of this paper).

It is obvious that the basic PAS scheme is worse than the low-complexity CAS in terms of both security and usability. Actually, even the above comparison is not a fair one, either, since not all security and usability factors are considered. In our opinion, comparing performance of different human authentication systems is not an easy task without a comprehensive security and usability study of all the systems involved. But one principle is clear: the comparison of usability should be made for the same level of security against various kinds of attacks, and vice versa. In other words, the performance comparison should be done by considering both security and usability simultaneously.

Another problem with the basic PAS scheme is that it requires too long passwords. For the default setting, each user has to remember two cell indices and two words of length 10 . In total there are 4 digits and 20 characters to be remembered. Although there are several ways of creating easily memorable but still strong passwords as discussed in [3], we doubt if they indeed work in reality for average users. In [3] it was not reported if the participants in the user study had difficulties choosing their passwords and how likely they might forget their passwords. According to a largescale user study on web password habits [20], the average password length is around 6 to 9 and passwords longer than 13 characters are rare. Hence, it remains a question if 4 
digits plus 20 characters are indeed usable.

In case the usability of the basic PAS scheme may be a problem, the extended PAS scheme seems even more difficult for average users to handle. Even when $k=2$, the average login time will be at least doubled, which is about $2 \times 84$ seconds $\approx 2.8$ minutes, exceeding the upper bound of more than half of the average users according to the user study reported in [3]. In addition, if the value of len remains the same, the number of digits and characters to be remembered will also be doubled. By using a smaller value of len, the memorability problem can be relaxed, but it has no obvious influence on the average login time, which does not depend on the value of len. Further more, we expect the error rate will also significantly increase due to the added complexity of handling more terms in each predicate.

To sum up, although we cannot definitely say if the basic PAS scheme is usable or not, it is clear that the extended PAS scheme is not usable. Because of this, in the next section we will focus our attention mainly on the basic PAS scheme.

\section{A Probabilistic Attack}

The security analysis given in the previous section has shown that security of the PAS scheme is much weaker than claimed in [3]. In Section IV-A, we also show that the number of candidate predicates decreases exponentially as $t$ increases. For the default setting of the basic PAS scheme, the predicate pair used can be uniquely determined with high probability when $t=2$, since $1+\left((25 \times 26)^{2}-1\right) / 2^{2 \times 5 \times 2} \approx$ $1.4029<2$. This leads to partial breaking of the password. To avoid information leakage from the observed responses, $t_{\max }=1$ is suggested in [3]. With this setting, on average one will get $1+\left((25 \times 26)^{2}-1\right) / 2^{2 \times 5} \approx 413.6$ predicate pairs for each observed session. Since the predicate pairs used for different authentication sessions are different, it seems impossible to break the password when $t_{\max }=1$.

In this section, we propose a probabilistic attack that is still able to partially break the password even when $t_{\max }=1$. The key point is that the same set of cell indices appear in the $p$-predicate vectors used for all authentication sessions. This makes it possible to further exploit the correlation among different $p$-predicate vectors to get more information about the secret cell indices, which can then be used to further refine the set of candidate $p$-predicate vectors obtained from each observed authentication session. When the number of observed authentication sessions is large enough, we may be able to uniquely determine the cell indices. The probabilistic nature of the attack allows us to guess the cell indices even when the number of observed authentication sessions is not enough. After determining the cell indices, some secret characters may also be uniquely determined or there are only a few candidates left.

The success rate of the attack smoothly increases as the number of observed authentication sessions increases. For the default setting of the basic PAS scheme, experimental results show that only 7 observed authentication sessions are enough to achieve a success rate higher than $50 \%$, which refutes the claim that the password can be used for at least 10 times before renewal. Even with only two observed authentication sessions, the success rate is not negligible - around $3.5 \%$. The probabilistic attack is also computationally efficient. Its maximal complexity is always strictly smaller than the complexity of the brute force attack.

In the following, we describe how the attack works, and give some theoretical analyses on the probabilities involved and the computational complexity of the attack. Experimental results are given to demonstrate the feasibility of the proposed attack on the default setting of the basic PAS scheme. Finally, we show the consequence of breaking the secret cell indices is that the PAS scheme is downgraded to a challenge-response protocol working like a one-time password (OTP) system but with worse usability and security.

\section{A. Description of the Attack}

To simplify the description of the probabilistic attack, we show how it works for the basic PAS scheme when the attacker knows the value of len. In this case, given $\hat{t} \geq 1$ observed authentication session(s), a step-by-step description of the probabilistic attack is as follows:

- Step 1: For each observed authentication session, obtain a set of $p$-predicate vectors agreeing with all the $n_{r}$ challenge-response pairs. Denote all the $\hat{t}$ sets by $\mathbb{P}_{i}$, $i=1, \ldots, \hat{t}$.

- Step 2a: For each $p$-predicate vector $\left(\operatorname{Pred}_{1}, \ldots, \operatorname{Pred}_{p}\right)$ in $\mathbb{P}_{i}$, extract the cell-index part to get a $p$-tuple cellindex vector $\left(c_{1}, \cdots, c_{p}\right)$. All the $p$-tuple cell-index vectors form a new set $\mathbb{C}_{i}$.

- Step 2b: Calculate $\mathbb{C}^{*}=\bigcap_{i=1}^{\hat{t}} \mathbb{C}_{i}$.

- Step 2c: Use $\mathbb{C}^{*}$ to refine each set $\mathbb{P}_{i}$ and get a new set as follows: $\mathbb{P}_{i}^{*}=\left\{x=\left(c_{i}, h_{i}\right) \mid x \in \mathbb{P}_{i} \wedge c_{i} \in \mathbb{C}^{*}\right\}$.

- Step 3a: If $\left|\mathbb{C}^{*}\right|=1$, all the $p$ secret cell indices can be immediately determined, and thus some candidates of those secret characters in $\mathbb{P}_{i}^{*}$ corresponding to the secret cell indices can also be obtained.

- Step $3 b$ : If $\left|\mathbb{C}^{*}\right|>1$, count the number of times each cell-index vector occurs in $\mathbb{P}_{1}^{*}, \ldots, \mathbb{P}_{\hat{t}}^{*}$ and rank the cell-index vectors in order of occurrence. All cell-index vectors that are ranked first are the candidates for the secret cell-index vector. All characters in $\mathbb{P}_{1}^{*}, \ldots, \mathbb{P}_{\hat{t}}^{*}$ that correspond to these candidates cell-index vectors are then the candidates for the secret characters.

In the proposed attack, Step 1 corresponds to the brute force attack targeting each $p$-predicate vector, and Step 2 exploits the correlation existing between different $p$ predicate vectors (i.e., the static cell-index vector). Step 3 has two different cases, according to the cardinality of $\mathbb{C}^{*}$. The ranking based strategy in Step $3 b$ is justified by the 
Table V

The RANGe OF $\hat{t}$ TO ENSURE $\operatorname{Pr}\left[\left|\mathbb{C}^{*}\right|=1\right] \geq q$.

\begin{tabular}{c||c|c|c|c|c|c|c|c|c|c|c}
\hline$q$ & 0.01 & 0.05 & 0.1 & 0.2 & 0.3 & 0.4 & 0.5 & 0.6 & 0.7 & 0.8 & 0.9 \\
\hline$\hat{t} \geq$ & 7 & 8 & 8 & 9 & 9 & 9 & 10 & 10 & 11 & 11 & 12 \\
\hline
\end{tabular}

fact that the secret cell-index vector appears most frequently, since it occurs at least once while others may never occur. A more detailed analysis on this ranking probability will be discussed in Section V-B2. Step 3b is the main part to make the attack work in a probabilistic manner.

\section{B. Theoretical Analysis}

In this subsection, we show some theoretical analyses on Steps $3 a$ and $3 b$ of the attack.

\section{1) Number of observed authentication sessions}

First let us investigate how many observed authentication sessions will ensure that $\left|\mathbb{C}^{*}\right|=1$ happens with high probability. According to our discussion in Section IV-A, the probability that each incorrect $p$-predicate vector will remain in $\mathbb{P}_{i}$ is $1 / 2^{l n_{r}}$. Then, we can derive $\operatorname{Pr}\left[\left|\mathbb{P}_{i}\right|=a+1\right]=$ $\left(\begin{array}{c}N_{1} \\ a\end{array}\right)\left(1 / 2^{l n_{r}}\right)^{a}\left(1-1 / 2^{l n_{r}}\right)^{N_{1}-a}$, where $0 \leq a \leq N_{1}$ and $N_{1}=(M H)^{p}-1$. Note that the correct $p$-predicate vector is always in $\mathbb{P}_{i}$, so $\left|\mathbb{P}_{i}\right| \geq 1$.

Given a set $\mathbb{P}_{i}$ of size $a+1$, let us estimate the probability that an incorrect $p$-tuple cell-index vector $\left(c_{1}, \ldots, c_{p}\right)$ belongs to $\mathbb{C}_{i}$ under the assumption that all incorrect $p$ predicate vectors appear in $\mathbb{P}_{i}$ with equal probability. To facilitate the following discussion, denote the probability by $\rho_{0}(a)$. When $a>N_{1}-H^{p}$, we can see $\rho_{0}(a)=1$, since there can be a maximum of $N_{1}-H^{p} p$-predicate vectors with other cell-index vectors. When $a \leq N_{1}-H^{p}$, the probability is $\rho_{0}(a)=1-\left(\begin{array}{c}N_{1}-H^{p} \\ a\end{array}\right) /\left(\begin{array}{c}N_{1} \\ a\end{array}\right)=1-\prod_{i=0}^{a-1}\left(1-\frac{H^{p}}{N_{1}-i}\right)$.

Based on the above results, for a randomly generated set $\mathbb{P}_{i}$ whose size is unknown, the probability that an incorrect cell-index vector $\left(c_{1}, \ldots, c_{p}\right)$ belongs to $\mathbb{C}_{i}$ is as follows

$$
\begin{aligned}
\rho & =\operatorname{Pr}\left[\left(c_{1}, \ldots, c_{p}\right) \in \mathbb{C}_{i}\right] \\
& =\sum_{a=0}^{N_{1}} \rho_{0}(a) \cdot \operatorname{Pr}\left[\left|\mathbb{P}_{i}\right|=a+1\right] .
\end{aligned}
$$

Assuming the above probability $\rho$ does not depend on the subscript $i$, we get $\operatorname{Pr}\left[\left(c_{1}, \ldots, c_{p}\right) \in \mathbb{C}^{*}\right]=$ $\prod_{i=1}^{\hat{t}} \operatorname{Pr}\left[\left(c_{1}, \ldots, c_{p}\right) \in \mathbb{C}_{i}\right]=\rho^{\hat{t}}$. Then, we can further derive the probability that $\left|\mathbb{C}^{*}\right|=1$ as the probability that none of the $M^{p}-1$ incorrect cell-index vectors is in $\mathbb{C}^{*}$ : $\operatorname{Pr}\left[\left|\mathbb{C}^{*}\right|=1\right]=\left(1-\rho^{\hat{t}}\right)^{M^{p}-1}$. Let $\operatorname{Pr}\left[\left|\mathbb{C}^{*}\right|=1\right] \geq q$, we get the following condition: $\hat{t} \geq\left[\log _{\rho}\left(1-q^{\frac{1}{M^{p}-1}}\right)\right]$.

Once the parameters of the basic PAS scheme are all given, one can immediately estimate the value of $\rho$ and then calculate the minimal value of $\hat{t}$ corresponding to any threshold probability $q$. For the default parameters, $\rho=0.4834$. Table $\mathrm{V}$ shows the minimal value of $\hat{t}$ ensuring
$\left|\mathbb{C}^{*}\right|=1$ happens with different threshold probabilities $q$. We can see that 10 observed authentication sessions are enough to guarantee $q \geq 0.5$.

\section{2) Ranking Probability in Step $3 b$}

Table $\mathrm{V}$ shows that Step $3 \mathrm{a}$ is not able to effectively reduce the number of observed authentication sessions. When $q=0.5$, we need 10 observed authentication sessions, which is the maximal number before password renewal. Although we may be able to break the password with 7 observed authentication sessions, the probability is too low. Step $3 b$ can help the attack work with even less than 7 observed authentication sessions, and with a nontrivial success rate.

To make a theoretical analysis on the ranking probability problem involved in Step 3b, we need to estimate the size of $\mathbb{P}_{i}^{*}$. Assuming the number of incorrect $p$-predicate vectors in $\mathbb{P}_{i}$ decreases with the same rate as the number of incorrect cell-index vectors in $\mathbb{C}$, we have $\left|\mathbb{P}_{i}^{*}\right|=1+\rho^{\hat{t}}\left(\left|\mathbb{P}_{i}\right|-1\right)$. Since $E\left(\left|\mathbb{P}_{i}\right|\right)=1+N_{1} / 2^{l n_{r}}$, we get $E\left(\left|\mathbb{P}_{i}^{*}\right|\right)=1+\rho^{\hat{t}} N_{1} / 2^{l n_{r}}$.

With the estimation of $\left|\mathbb{P}_{i}^{*}\right|$, we need to know the probability that the correct cell-index is among the most frequent one(s). This is equivalent to the following problem.

There are $N=M^{p}$ types of objects. Type-1 objects occur with probability $q_{1}=\left(H^{p}-1\right) / N_{1}$, and all other objects occur with probability $q_{0}=$ $H^{p} / N_{1}$. Randomly pick $L=\sum_{i=1}^{\hat{t}}\left(\left|\mathbb{P}_{i}^{*}\right|-1\right)$ objects with the above probabilities and add $\hat{t}$ more type-1 object(s), what is the probability that the number of type-1 object(s) is not less than the number of objects of any other type?

Note that $q_{1}+(N-1) q_{0}=1$ for the above problem. To facilitate our discussion, denote the number of type- $i$ objects in the $L$ objects by $\#\left(O_{i}\right)$. It is not easy to get an explicit solution to the above problem. Now let us try to derive a practical lower bound of the probability. When $L \leq \hat{t}, \#\left(O_{i}\right) \leq L \leq \hat{t} \leq \#\left(O_{1}\right)+\hat{t}$ always holds, so $\operatorname{Pr}\left[\max _{i=2}^{N}\left(\#\left(\bar{O}_{i}\right)\right) \leq \#\left(\bar{O}_{1}\right)+\hat{t}\right]=1$. When $L \geq \hat{t}+1$, we have the following result:

$$
\begin{aligned}
& \operatorname{Pr}\left[\max _{i=2}^{N}\left(\#\left(O_{i}\right)\right) \leq \#\left(O_{1}\right)+\hat{t}\right] \\
= & 1-\operatorname{Pr}\left[\exists i \in\{2, \ldots, N\}, \#\left(O_{i}\right)>\#\left(O_{1}\right)+\hat{t}\right] \\
\geq & 1-\min \left(1, \sum_{i=2}^{N} \operatorname{Pr}\left[\#\left(O_{i}\right) \geq \hat{t}+1\right]\right) \\
= & 1-\min \left(1,(N-1) \sum_{i=\hat{t}+1}^{L}\left(\begin{array}{c}
L \\
i
\end{array}\right) q_{0}^{i}\left(1-q_{0}\right)^{L-i}\right) .
\end{aligned}
$$

When $\hat{t}$ is close to 1 , the above lower bound is generally equal to 0 . But as $\hat{t}$ becomes larger, the lower bound quickly converges to 1 . Taking the default parameters and assuming $L=E\left(\sum_{i=1}^{\hat{t}}\left(\left|\mathbb{P}^{*}\right|-1\right)\right)=\hat{t} \rho^{\hat{t}} N_{1} / 2^{l n_{r}}$, we calculated the above lower bound for $\hat{t}=1, \ldots, 10$. For each value of $\hat{t}$, 
Table VI

$\operatorname{Pr}\left[\max _{i=2}^{N}\left(\#\left(O_{i}\right)\right) \leq \#\left(O_{1}\right)+\hat{t}\right]:$ THEORETICAL BOUND AND EXPERIMENTAL ESTIMATION.

\begin{tabular}{c||c|c|c|c|c}
\hline$\hat{t}$ & 1 & 2 & 3 & 4 & $\geq 5$ \\
\hline Theoretical lower bound & 0 & 0 & 0.9473 & 0.9997 & 1 \\
\hline Experimental estimation & 0.0504 & 0.2915 & 0.9604 & 0.9999 & 1 \\
\hline
\end{tabular}

Table VII

$E\left(N_{\max }\right)$ : THEORETICAL BOUND AND EXPERIMENTAL ESTIMATION.

\begin{tabular}{c||c|c|c|c|c|c}
\hline$\hat{t}$ & 1 & 2 & 3 & 4 & 5 & $\geq 6$ \\
\hline Theoretical upper bound & 625 & 625 & 607.1 & 6.842 & 1.012 & 1 \\
\hline Experimental estimation & 3.6846 & 3.6184 & 1.7168 & 1.0086 & 1 & 1 \\
\hline
\end{tabular}

10000 random experiments were made to see how large the real probabilities are. Table VI shows the results.

Following a similar argument, we can also get:

$$
\begin{aligned}
& \operatorname{Pr}\left[\exists i \in\{2, \ldots, N\}, \#\left(O_{i}\right) \geq \#\left(O_{1}\right)+\hat{t}\right] \leq \\
& \quad \min \left(1,(N-1) \sum_{i=\hat{t}}^{L}\left(\begin{array}{c}
L \\
i
\end{array}\right) q_{0}^{i}\left(1-q_{0}\right)^{L-i}\right) .
\end{aligned}
$$

Then, assuming there are $N_{\max }$ cell-index vectors occurring most often in $\mathbb{P}_{i}^{*}, \ldots, \mathbb{P}_{\hat{t}}^{*}$, i.e., $N_{\max }$ is the cardinality of the set $\left\{i \mid \#\left(O_{i}\right)=\max _{j=1}^{M^{p}} \#\left(O_{j}\right)\right\}$, we can get an upper bound of its mean: $E\left(N_{\max }\right) \leq 1+\left(M^{p}-1\right)$. $\min \left(1,(N-1) \sum_{i=\hat{t}}^{L}\left(\begin{array}{c}L \\ i\end{array}\right) q_{0}^{i}\left(1-q_{0}\right)^{L-i}\right)$. For the default setting of the PAS scheme and $\hat{t}=1, \ldots, 10$, Table VII shows the theoretical upper bound and the real value of $E\left(N_{\max }\right)$ estimated from 10000 random experiments.

The data in Tables VI and VII imply that one can recover the secret cell-index vector with high probability with only 3 observed authentication sessions.

\section{Complexity Analysis}

The computational complexity of the proposed probabilistic attack is the sum of the complexity of all the three steps. The complexity of Step 1 is $\hat{t}(M H)^{p}$, which is the maximal number of $p$-predicate vectors one has to check for all the $\hat{t}$ observed authentication sessions to get $\mathbb{P}_{i}$. After Step 1 is finished, the average size of each $\mathbb{P}_{i}$ is $1+N_{1} / 2^{l n_{r}}$, so the average complexity of Step 2 is $\hat{t}\left(1+N_{1} / 2^{l n_{r}}\right)$. The complexity of Step 3a is very small, so it can be omitted. The ranking done in Step $3 b$ has a complexity $\sum_{i=1}^{\hat{t}}\left|\mathbb{P}_{i}^{*}\right|=\hat{t}\left(1+\rho^{\hat{t}} N_{1} / 2^{l n_{r}}\right)$. The worst-case complexities of Step 2 and $3 b$ are always less than the complexity of Step 1. As a whole, we can see the overall complexity of the attack is determined by Step 1, which has an upper bound $O\left(\hat{t}(M H)^{p}\right)$. For the default setting of the PAS basic scheme and $\hat{t}=4$, the complexity is $O\left(\hat{t}(M H)^{p}\right)=O\left(2^{20.7}\right)$.

Since the size of the password space of the basic PAS scheme is len $\cdot(M H)^{p}$, the complexity of the probabilistic attack is always strictly smaller than that of the brute force attack since $\hat{t}<$ len always holds.
Table VIII

THE SUCCESS RATE OF BREAKING THE SECRET CELL-INDEX VECTOR AND THE NUMBER OF CANDIDATES ESTIMATED FROM REAL ATTACKS.

\begin{tabular}{c||c|c|c|c|c|c|c|c|c|c}
\hline$\hat{t}$ & 1 & 2 & 3 & 4 & 5 & 6 & 7 & 8 & 9 & 10 \\
\hline Success rate & 0.012 & 0.035 & 0.071 & 0.13 & 0.24 & 0.41 & 0.60 & 0.76 & 0.86 & 0.94 \\
\hline Number & 3.01 & 2.51 & 2.02 & 1.73 & 1.51 & 1.36 & 1.23 & 1.10 & 1.03 & 1.01 \\
\hline
\end{tabular}

\section{Experimental Results}

Based on the above theoretical analysis and the complexity estimation, we can see the attack is feasible as long as $(M H)^{p}$ is not cryptographically large. This condition is satisfied for the default setting of the PAS scheme.

We developed a MATLAB implementation of the basic PAS scheme with $p=2$, and tested the real performance of the proposed probabilistic attack. On a PC with a $2.4 \mathrm{GHz}$ Intel Core2 Duo CPU and 2GB memory, one successful attack with $\hat{t}$ observed authentication sessions consumes only $5 \hat{t}$ seconds. The MATLAB code is available at http: //www.hooklee.com/Papers/Data/PAS.zip.

The statistical results of 1000 real attacks targeting the default setting of the basic PAS scheme are shown in Table VIII. It turned out that the real performance is worse than the theoretical analysis obtained in Section V-B2. We attribute this to the deviation of real attacks from some of the theoretical assumptions we made in the theoretical analysis in Section V-B2. For instance, we calculate the values in Table VI by assuming $E\left(\left|\mathbb{P}_{i}^{*}\right|\right)=1+\rho^{\hat{t}} N_{1} / 2^{l n_{r}}$ and $L=\hat{t} \rho^{\hat{t}} N_{1} / 2^{l n_{r}}$, but in practice their values vary in a wide range around the means. Despite the mismatch between Table VIII and Table VI, we can see the success rate of breaking the secret cell-index pair and the average number of candidates follow the same pattern as the data in Table VII.

The data in Table VIII show that 7 observed authentication sessions are enough to break the secret cell-index pair with probability greater than $50 \%$. Even with only two observed authentication sessions, the success rate is high enough $(3.5 \%)$ to threaten a considerable percentage of users.

\section{E. Consequences of the Probabilistic Attack}

Note that it is impossible and unnecessary to break the whole password with the probabilistic attack, since some secret characters will never occur until the last authentication session. In fact, the main consequence of breaking the secret cell indices is the following: the password becomes a set of len words $\left\{W_{j}^{*}=w_{1}[j] \cdots w_{p}[j]\right\}_{j=1}^{l e n}$, each of which is used for exactly one authentication session. After all the len words $\left\{W_{j}^{*}\right\}_{j=1}^{l e n}$ are used up, a new password have to be issued to the user. Clearly, this means PAS now works essentially like a one-time password (OTP) system, where each word $W_{j}^{*}$ is the OTP used for each authentication session and expires immediately after being used.

The degradation of the PAS scheme to an OTP-like system has several consequences. First, this fact disqualifies PAS 
as a better solution than common OTP systems. Second, the downgraded PAS scheme is still a challenge-response protocol, which asks the user to go through the same process as in the original PAS scheme. In comparison, common OTP systems are not based on a challenge-response structure and the user is simply asked to input the dynamic password in an input box, so the usability is much better. Third, the downgraded PAS scheme offers a lower security against random guess attack. We can derive that the success rate of the downgraded PAS scheme becomes

$$
\frac{1}{2^{\ln _{r}}}+\frac{2^{l n_{r}}-1}{2^{\ln _{r}\left(\begin{array}{c}
H+k-1 \\
k
\end{array}\right)^{p}}}
$$

Comparing the above equation with Eq. (1), we can see the success rate becomes larger. For the default parameters, Eq. (1) is around $9.7893 \times 10^{-4}$, but Eq. (5) is around $2.4544 \times 10^{-3}$, nearly 2.5 times larger. To maintain the same level of security, the parameters have to be increased accordingly, which will make usability even worse.

\section{Conclusion}

In this paper, we re-evaluate the security of the predicatebased authentication service (PAS) proposed in [3]. We show that PAS is insecure against both brute force attack and a probabilistic attack. The probabilistic attack can break part of the password even with a small number of observed authentication sessions. The breaking of part of the password downgrades PAS to an OTP-like system, thus nullifying its main advantages over common OTP systems.

It is possible to enhance security of PAS by increasing the values of some parameters, unfortunately, which will decrease usability and make the system not useful as a practical solution. This problem about curse of usability is the main reason why it is very difficult to design a both secure and usable authentication system secure against passive adversaries who can observe all authentication sessions.

\section{Acknowledgments}

Shujun Li was supported by a fellowship from the Zukunftskolleg of the University of Konstanz, Germany, which is part of the "Excellence Initiative" Program of the DFG (German Research Foundation). Hassan Jameel Asghar was supported by an MQRES (Macquarie University Research Excellence Scholarships) International PhD Scholarship. Josef Pieprzyk was supported by the Australia Research Council under Grant DP0987734. Ahmad-Reza Sadeghi was supported by the EU project CACE (Computer Aided Cryptography Engineering, http://www.cace-project.eu). Huaxiong Wang was supported by the National Research Foundation of Singapore under Research Grant NRF-CRP2-2007-03 and the Singapore Ministry of Education under Research Grant T206B2204.

\section{References}

[1] T. Matsumoto and H. Imai. Human identification through insecure channel. In Advances in Cryptology - EUROCRYPT'91, volume 547 of $L N C S$, pages 409-421. SpringerVerlag, 1991.

[2] S. Li and H.-Y. Shum. Secure human-computer identification against peeping attacks (SecHCI): A survey. http://www. hooklee.com/Papers/SecHCI-Survey.pdf, 2003.

[3] X. Bai et al. PAS: predicate-based authentication services against powerful passive adversaries. In Proc. ACSAC'2008, pages 433-442. IEEE Computer Society, 2008.

[4] C.-H. Wang, T. Hwang, and J.-J. Tsai. On the Matsumoto and Imai's human identification scheme. In Advances in Cryptology - EUROCRYPT'95, volume 921 of LNCS, pages 382-392. Springer-Verlag, 1995.

[5] T. Matsumoto. Human-computer cryptography: An attempt. In Proc. ACM CCS'96, pages 68-75. ACM, 1996.

[6] N. J. Hopper and M. Blum. Secure human identification protocols. In Advances in Cryptology - ASIACRYPT 2001, volume 2248 of $L N C S$, pages 52-66. Springer-Verlag, 2001.

[7] X.-Y. Li and S.-H. Teng. Practical human-machine identification over insecure channels. J. Combinatorial Optimization, 3(4):347-361, 1999.

[8] S. Li and H.-Y. Shum. Secure human-computer identification (interface) systems against peeping attacks: SecHCI. IACR's Cryptology ePrint Archive: Report 2005/268, 2005.

[9] H. Jameel, R. Shaikh, H. Lee, and S. Lee. Human identification through image evaluation using secret predicates. In Topics in Cryptology - CT-RSA 2007, volume 4377 of LNCS, pages 67-84. Springer-Verlag, 2007.

[10] Hassan Jameel et al. Image-feature based human identification protocols on limited display devices. In Information Security Applications (WISA'2008), volume 5379 of LNCS, pages 211-224. Springer-Verlag, 2009.

[11] D. Weinshall. Cognitive authentication schemes safe against spyware. In Proc. IEEE S\&P'2006, pages 295-300. IEEE Computer Society, 2006.

[12] P. Golle and D. Wagner. Cryptanalysis of a cognitive authentication scheme. In Proc. IEEE S\&P'2007, pages 6670. IEEE Computer Society, 2007.

[13] R. Dhamija and A. Perrig. Déjà Vu: A user study using images for authentication. In Proc. 9th USENIX Security Symposium, pages 45-58. USENIX, 2000.

[14] V. Roth, K. Richter, and R. Freidinger. A PIN-entry method resilient against shoulder surfing. In Proc. ACM CCS'2004, pages 236-245. ACM, 2004.

[15] D. Lin, P. Dunphy, P. Olivier, and J. Yan. Graphical passwords \& qualitative spatial relations. In Proc. SOUPS'2007, pages 161-162. ACM, 2007.

[16] H. Zhao and X. Li. S3PAS: A scalable shoulder-surfing resistant textual-graphical password authentication scheme. In Proc. AINAW'2007, volume 2, pages 467-472, 2007.

[17] E. Hayashi, R. Dhamija, N. Christin, and A. Perrig. Use Your Illusion: Secure authentication usable anywhere. In Proc. SOUPS'2008, pages 35-45. ACM, 2008.

[18] A. De Luca and B. Frauendienst. A privacy-respectful input method for public terminals. In Proc. NordiCHI'2008, pages 455-458. ACM, 2008.

[19] H. Sasamoto, N. Christin, and E. Hayashi. Undercover: Authentication usable in front of prying eyes. In Proc. ACM CHI'2008, pages 183-192. ACM, 2008.

[20] D. Florêncio and C. Herley. A large-scale study of web password habits. In Proc. WWW'2007, pages 657-665, 2007. 